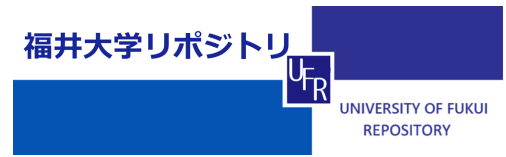

Sel $f$-di sper si on of mercury net al i nt o aqueous sol ut i ons

\begin{tabular}{|l|l|}
\hline 著者 & Aoki K, Li C., N shi umi T. , Chen J. \\
\hline $\begin{array}{l}\text { j our nal or } \\
\text { publ i cat i on ti t l e }\end{array}$ & Journal of El ect r oanal yt i cal Chemi st ry \\
\hline vol une & 682 \\
\hline page r ange & $66-71$ \\
\hline year & $2012-08$ \\
\hline URL & ht t p: //hdl . handl e. net /10098/9867 \\
\hline
\end{tabular}




\title{
Self-dispersion of mercury metal into aqueous solutions
}

\author{
Koichi Aoki*, Chunyan Li, Toyohiko Nishiumi, Jingyuan Chen \\ Department of Applied Physics, University of Fukui, 3-9-1 Bunkyo, Fukui, 910-0017 Japan
}

\begin{abstract}
Mercury metal dispersed spontaneously into the aqueous solution as micro-droplets when it came in quiescent contact with the solution. The self-dispersion was observed by means of the dynamic light scattering method, thermogravimetry and voltammetry. The mercury microdroplets generated in water had ca. $0.3 \mu \mathrm{m}$ in diameter. The diameter and the concentration of the droplets were stable. The concentration of mercury might be 1-3 $\mu \mathrm{mol} \mathrm{dm} \mathrm{dm}^{-3}$ if the droplets were to be dissolved homogeneously in water. The concentration exceeds the environmental limits by three orders of magnitude. The dispersed mercury was confirmed not to be ions or oxide of mercury but to be metal droplets. The self-dispersion of mercury belongs to the self-emulsification at liquid|liquid interfaces without surfactants.
\end{abstract}

key words: micro-droplets of mercury; self-emulsification; dynamic light scattering; stripping voltammetry

Corresponding author, Tel: +81 77627 8665, fax: +81 776278494

E-mail: kaoki@u-fukui.ac.jp 


\section{Introduction}

A mixture of two solvents takes either state, a mutually dissolved state in molecular form or a dispersed state in droplet form. The former has enabled the thermodynamic analysis of ion-transfer-voltammetric data [1-3]. On the other hand, the latter is so heterogeneous in emulsion form that thermodynamic analysis is complicated. It is sometimes formed spontaneously when oil comes in contact with water phase quiescently [4-10]. Emulsification makes electrochemical responses delayed [11-14], fluctuated [15,16], pulsed [17], and convective unexpectedly [7]. These responses are not irreproducible, because size and the number concentrations of droplets are not controlled.

Self-emulsion, which is spontaneous dispersion of liquid into continuous liquids, can be generalized to self-colloid formation such as self-formation of foams, aerosols gels. We have found that hydrogen gas was stabilized in aqueous solution as micro-bubbles with a long life [18]. If this concept is applied to mercury|water interfaces, mercury droplets may also be dispersed in water phase. This possibility can be inferred from the polarographic behavior which often causes convection to yield polarographic maximum currents [19]. The self-induced convection has been observed as local velocity profiles $[20,21]$. Convection near the mercury electrode may disturb electrde|solution interfaces as if the interfaces were to be mixed vigorously. Droplets caused by the interfacial convection has been observed at the oil|water interface [15]. The dispersion of mercury in water has also been recognized in the fields of environmental chemistry [22,23], instrumental analytical techniques [24,25] and public health [26-28]. The concentrations of the dispersion have been reported to range from 0.02 to $5 \mu \mathrm{mol} \mathrm{dm}^{-3}$, depending not only on measurement techniques but also on conditions of solutions. They may contain not only mercury droplets but also mercury ions, oxides, halides and organomercury. 
Theoretical work of emulsions including formation and coalescence has been reported by Lifshitz and Slyozov [29], and dependently by Wagner [30,31]. The concept is based on the dynamics in which an enhancement of the surface energy by formation of droplets causes diffusion of droplets to coalescence one another. It is, however, important to discuss thermodynamic stability of emulsions rather than the dynamics. The stability of emulsion has recently been discussed in the light of the balance of surface energy of dispersed droplets with formation entropy by use of statistical mechanics [32]. This theory mentions that the smallest and the largest droplets are preferentially formed in the number, and that the largest droplets contribute mostly to analytical detection of droplets. On this prediction, we demonstrate here the presence of stable droplets of mercury metal which are generated spontaneously at the mercury|water interface. Our techniques of the detection of the droplets are the dynamic light scattering method, thermogravimetry and voltammetry.

\section{Experimental}

Mercury metal (Nacalai Tesque, Kyoto) used was within 6 months after purchase. Mercury being in long storage showed irreproducible results, probably because of degradation of mercury by water or oxygen. Mercuric perchlorate (Strem Chemicals, MA) was used as received. Hydrochloric acid and sodium chloride were of analytical grade. Water was deionized and distilled.

A small amount of mercury was collected with a $1 \mathrm{~cm}^{3}$ dried injector. When mercury was left in water for a period longer than a half day, floating substance was gradually recognized on the water surface. In order to avoid the oxidation, air was purged from solution with nitrogen gas. Mercury in the solution was kept in nitrogen atmosphere. 
An aqueous suspension of mercury was prepared by bubbling nitrogen gas into 200 $\mathrm{cm}^{3} 0.1 \mathrm{M} \mathrm{HClO}_{4}+0.05 \mathrm{M} \mathrm{NaCl}$ aqueous solution for $30 \mathrm{~min}$, adding a mercury drop $(0.04 \mathrm{~g})$ into the solution, and applying ultrasonication to the solution under nitrogen atmosphere for 30-50 min. Then the solution turned turbid at the expense of the mercury drop.

The working electrodes were a gold disk $1.6 \mathrm{~mm}$ in diameter and a platinum wire $0.5 \mathrm{~mm}$ in diameter. The reference electrode was a saturated calomel electrode (SCE). The counter electrode was a platinum coil. The surface of the gold electrode was renewed by grinding it with abrasive paper and then polishing it with alumina powder.

The potentiostat used was Compactstat (Ivium, Netherlands). Electrochemical conditions of square-wave voltammetry were $10 \mathrm{mM}$ pulse width, $150 \mathrm{~Hz}$ frequency, 2 $\mathrm{mV}$ potential step width. The size distribution was determined by a dynamic light scattering (DLS) instrument (Malvern Zetasizer Nano-ZS, UK). Thermogravimetry (TG) was carried out with Thermo Plus, TG8120 (Rigaku, Tokyo).

The sample container of TG was an alumina pan with inner volume $0.03 \mathrm{~cm}^{3}$ and $84 \mathrm{mg}$ in weight. Our aim of using TG was to determine weight of mercury selectively against water through time-dependent amount of evaporation. The temperature was controlled to be $40^{\circ} \mathrm{C}$, which may evaporate water gradually. A sample in TG was weighed in nitrogen flow of $50 \mathrm{~cm}^{3} \mathrm{~min}^{-1}$. Accuracy of weight under these conditions was $\pm 1 \mu \mathrm{g}$. The weight of the wet alumina pan in the TG instrument decreased for two hours linearly with the time by $30 \mathrm{mg}$, and kept a constant suddenly. This behavior indicates no absorption of water to the pan. TG was applied to a dry mercury drop 93 mg on the dry alumina pan. The weight decreased linearly with the time. The evaporation rate of mercury at $40^{\circ} \mathrm{C}$ was $0.0279 \mathrm{mg} / \mathrm{h}$ per $100 \mathrm{mg}$ mercury.

\section{Results and Discussion}




\subsection{Dynamic Light Scattering}

It is of interest to estimate size and a life of mercury droplets which are dispersed compulsorily into water by ultrasonication. A dry mercury drop $0.5 \mathrm{mg}$ was inserted into $50 \mathrm{~cm}^{3}$ deaerated distilled water, which was ultrasonicated for 30 min under nitrogen atmosphere. The resulting suspension was turbid and slightly grey without visible mercury drops. The turbidity should be ascribed to dispersed mercury droplets, the diameter of which should be close to or larger than visible light wavelength. DLS measurement was applied to the suspension a few hours after being left under nitrogen atmosphere. Various sizes of particles were presented in the suspension, as is shown in Fig. 1(a). Nitrogen gas bubbles in water also exhibited a distribution of particle (Fig. 1(b)). The distribution of mercury suspension was different from that of nitrogen gas. The intensity of light scattering, called kcps (kilo counts per second), for mercury suspension was $10^{5}$, whereas that for nitrogen gas was less than 100 . Therefore, the distribution in Fig. 1(a) should surely be caused by the mercury droplets. Figure 2 shows plots of diameters at the maximum distribution and kcps values against the time during which the suspension was left alone under nitrogen atmosphere. Droplets with diameters between 0.2 and $0.3 \mu \mathrm{m}$ were dispersed for a few hours without variation of kcps. The constant value of kcps suggests occurrence of neither coalescence nor sedimentation

In order to examine whether mercury is dispersed spontaneously in water, a dry mercury drop was inserted quiescently into the deaerated water with a syringe. The water including the mercury drop was left in nitrogen atmosphere for $13 \mathrm{~h}$ without mixing. A small volume of water over the mercury was collected with a pipette, and was applied to DLS. The DLS distribution was shown in Fig. 1(c), depicting the presence of particles $0.3 \mu \mathrm{m}$ in diameter. It can be discriminated against the gas bubbles (Fig. 1(b)). 
Values of kcps increased with the time of immersion of mercury. Therefore the particles were the spontaneously dispersed mercury droplets.

Dispersed mercury might be sparingly soluble particles of mercury oxide, which are generated by remaining oxygen. Mercury oxide is readily dissolved in strong acids to yield mercurous and mercuric ions. When perchloric acid was added to the suspension so that $\mathrm{pH}$ was 1 , the DLS showed the same as the DLS data for the neutral suspension. Therefore mercury oxide could not participate in the intensity of DLS.

A strategy of determining concentrations of dispersed mercury droplets is to use a calibration curve which relates the concentration with the intensity (kcps) of scattering light. In order to obtain the calibration, we prepared compulsively the dispersed mercury suspension from known amounts of mercury and water. Values of kcps were obtained for quantitatively diluted suspensions, and are showed in Fig. 3. An almost proportional relation between kcps and the concentration, $c$, was obtained at low concentrations (< $60 \mu \mathrm{M}$ ), as predicted. The small intercept (cannot be seen in Fig. 3) is due to nitrogen bubbles. In contrast, kcps-values for concentrations over $60 \mu \mathrm{M}$ were lower than the proportional line, probably because of a loss of incident light intensity by the turbidity of the suspension.

The approximately proportional line was used for the calibration curve of determining concentrations of spontaneously dispersed mercury. Mercury was left in water for a half day without mixing. Solution over the mercury was collected and its kcps value was obtained. From the calibration line, we determined the concentration, 3 $\mu \mathrm{M}$ or $0.6 \mathrm{mg} \mathrm{dm}^{-3}$. This concentration was much smaller than that of spontaneous dispersion of nitrobenzene $(15 \mathrm{mM})$ [9]. The smallness can be explained by the fact that the interfacial tension of mercury|water is by one order magnitude larger than that of nitrobenzene/water. Concentrations (kcps) and diameters did not vary largely with periods of immersion of mercury, as is shown in Fig. 4. The concentration of 
spontaneously dispersed mercury is by three orders magnitude larger than the environmental limit $0.0005 \mathrm{mg} \mathrm{dm}^{-3}$ of revers in Japan.

\subsection{Thermogravimetry}

According to the result in section 3.1 , a mercury drop inserted into $1 \mathrm{dm}^{3}$ water should be dispersed by $0.6 \mathrm{mg}$, which corresponds to $0.04 \mathrm{~mm}^{3}$. We attempted to measure the decrease in the volume of a mercury drop by $0.04 \mathrm{~mm}^{3}$, but found oppositely a slight increase in the volume by a microscope. Water may be inserted into mercury, at a cost of dispersion of mercury, as widely known in polarographic techniques. We tried to obtain time-dependence of mass of water + mercury drop by evaporation, instead of volume change. The two processes were employed: (a) time-variation of weight of a wet mercury drop, and (b) that of the mercury drop after spontaneous dispersion.

(a) A mercury drop which was dried in a desiccator was weighed (weight $W(\mathrm{Hg})$ ), ca. $93 \mathrm{mg}$ ). It was transferred on the alumina pan in which water was filled. Then the pan was mounted on the TG instrument at $40^{\circ} \mathrm{C}$, being weighed with the time in the flow of nitrogen gas. The time-variation of the weight is shown in Fig. 5. The weight decreased linearly with the time until $1850 \mathrm{~s}$, during which the heat flow was negative. The weight after $1850 \mathrm{~s}$ was kept constant suddenly and the heat flow vanished. These features indicate that the decrease in the weight should be ascribed to evaporation of water, and that the evaporation should be completed at 1850 s. Figure 6(a) shows conceptual diagrams, where $W$ means known weight, $w$ does unknown. The observed values are listed in Table 1. If all the water in the pan is evaporated, the weight should decrease by $W\left(\mathrm{H}_{2} \mathrm{O}\right)$. However, the observed decrement was smaller than $W\left(\mathrm{H}_{2} \mathrm{O}\right)$. A part of water was confined to the mercury. The decrement rate after $1850 \mathrm{~s}$ was 2.5 times larger than the evaporation rate of mercury $(0.028 \mathrm{mg} / \mathrm{h})$. The amount smaller 
than $W\left(\mathrm{H}_{2} \mathrm{O}\right)$ may be caused by confinement of water in the mercury. The weight of the confined water, $w\left(\mathrm{H}_{2} \mathrm{O}(\mathrm{Hg})\right)$, was 0.15 g per 100 g mercury.

(b) A technique of determining the concentration of spontaneously dispersed mercury by weight is to take a difference between the weight of dry mercury and the weight of mercury immersed into much water for a long time. A weighed dry mercury drop was mounted in the pan. The pan containing the mercury drop was immersed in 30 $\mathrm{cm}^{3}$ water for one day, during which a part of mercury would be dispersed. Then the pan including the wet mercury was mounted on the TG instrument. The time-variation of the weight at $40^{\circ} \mathrm{C}$ was similar to that in Fig. 5. The diagram of the weight change is shown in Fig. 6(b). Since the weight at $t=0$ includes extra water in the pan, $w\left(\mathrm{H}_{2} \mathrm{O}\right)$, and a loss of weight, $\Delta w(\mathrm{Hg})$, by one day's immersion, it is given by $W(\mathrm{Hg})-\Delta w(\mathrm{Hg})+w\left(\mathrm{H}_{2} \mathrm{O}\right)$. If water were to be evaporated completely, the weight might be $W(\mathrm{Hg})-\Delta w(\mathrm{Hg})$. However, water confined to mercury, $w\left(\mathrm{H}_{2} \mathrm{O}(\mathrm{Hg})\right)$, should make the measured weight be $W(\mathrm{Hg})-\Delta w(\mathrm{Hg})+w\left(\mathrm{H}_{2} \mathrm{O}(\mathrm{Hg})\right)$. The weight, $w\left(\mathrm{H}_{2} \mathrm{O}(\mathrm{Hg})\right)$, was calculated $(0.073 \mathrm{mg})$ from experiment (a) for the mercury weight used. Then we obtained $\Delta w(\mathrm{Hg})=0.033$ mg, which corresponds to $1 \mathrm{mg} / 1 \mathrm{dm}^{3} \mathrm{H}_{2} \mathrm{O}$ or $5 \mu \mathrm{M}$. This value is larger than that by DLS $(3 \mu \mathrm{M})$. The difference may be due to underestimation of $w\left(\mathrm{H}_{2} \mathrm{O}(\mathrm{Hg})\right)$ in process (b), because the immersion time of the mercury into water in process (b) was much longer than the period in process (a).

\subsection{Electrochemistry}

Dispersed mercury droplets seem to be detected simply by electrochemical oxidation of mercury. However, there are some difficulties in the detection, e.g. low concentration of droplets, ambiguous oxidation potential owing to wide distribution of droplet size and surface activity, and unclear mass transport of droplets to the electrode. In order to overcome these difficulties, we firstly detect self-dispersed droplets 
qualitatively by amalgamation on a gold electrode. We secondly determine the concentration of dispersed droplets qualitatively by anodic stripping voltammetry, where the concentration to be determined is not number concentration of droplets but is the concentration of mercury ions which generates from oxidation of the droplets. The stripping voltammetry is made in the forced dispersed mercury suspension and spontaneously dispersed one.

We try to detect qualitatively the spontaneously dispersed droplets in acid solution, $0.1 \mathrm{M} \mathrm{HClO}_{4}$ in order to avoid formation of mercury oxide. A mercury drop was put in the deaerated acid solution, into which the platinum wire was inserted, as is illustrated in Fig. 7. It was left for $1 \mathrm{~h}$ by applying potential $0.0 \mathrm{~V}$ through the platinum wire to keep the reduced state of the mercury. The gold electrode was mounted near the mercury to collect spontaneously dispersed droplets in the form of amalgam. Voltammogram was obtained at the gold disk electrode, showing (Fig. 8(a)) oxidation waves in the potential domain from 0.4 to $0.9 \mathrm{~V}$ vs. SCE, discriminated against the background (b). The anodic waves can be attributed to the oxidation of mercury metal because there was no reduced species except for mercury. The anodic wave in Fig. 8 demonstrates generation of mercury droplets in the solution, transportation from the mercury drop to the gold electrode, formation of amalgam, and electrochemical oxidation of the amalgam. The oxidation charge was close to the amount of mono-molecular layer.

Our aim is to obtain concentrations of spontaneously dispersed mercury from voltammograms. The suitable electrochemical technique is anodic stripping voltammetry combined with square-wave voltammetry because the concentration is low. Before making a calibration curve, we examined voltammetric behavior of mercury and mercuric ion. We applied $0.0 \mathrm{~V}$ vs. SCE at the gold electrode for $60 \mathrm{~s}$ in the solution $0.04 \mathrm{mM} \mathrm{Hg}\left(\mathrm{ClO}_{4}\right)_{2}+0.1 \mathrm{M} \mathrm{HClO}_{4}$ for the cathodic deposition, and then performed square-wave voltammetry. Once the stripping voltammetry was made, the electrode 
surface lost brightness. The electrode was polished before each voltammetric run. The square-wave voltammograms showed two waves in Fig. 9, the potentials of which shifted to the negative direction with the iterative runs. Peak or shoulder potentials of voltammograms (b-d) are close to the formal potentials of the following reactions:

$$
\begin{array}{ll}
2 \mathrm{Hg}^{2+}+2 \mathrm{e}^{-} \leftrightarrow \mathrm{Hg}_{2}{ }^{2+} & E^{\mathrm{o}}=0.67 \mathrm{~V} \text { vs. SCE } \\
\mathrm{Hg}_{2}{ }^{2+}+2 \mathrm{e}^{-} \leftrightarrow 2 \mathrm{Hg} & E^{\mathrm{o}}=0.55 \text { V vs. SCE }
\end{array}
$$

The shift increased with the period of letting the electrode alone. A possible reason for the shift is a leak of $\mathrm{Cl}^{-}$from the SCE, because $\mathrm{Cl}^{-}$yields precipitates of $\mathrm{Hg}_{2} \mathrm{Cl}_{2}$ on the electrode, which enhances deposition of mercury by the deposition. A strategy of preventing the shift is to generate soluble $\left(\mathrm{HgCl}_{4}\right)^{2-}$ complex by adding excess concentration of $\mathrm{Cl}^{-}$. From the stability constant of $\left(\mathrm{HgCl}_{4}\right)^{2-}, 5 \times 10^{15}$ [33], we noticed that the usage of $0.05 \mathrm{M} \mathrm{NaCl}$ made $\left(\mathrm{HgCl}_{4}\right)^{2-}$ be a predominant species of mercuric compounds. The square-wave voltammogram in solution of $0.04 \mathrm{mM} \mathrm{Hg}\left(\mathrm{ClO}_{4}\right)_{2}+0.1$ $\mathrm{M} \mathrm{HClO}_{4}+0.05 \mathrm{M} \mathrm{NaCl}$ (Fig. 9(e)) did not show any time dependence.

Anodic stripping voltammetry was made in the above solution into which mercury was dispersed by ultrasonication. The square-wave voltammogram after the cathodic deposition, as shown in Fig. 10(a), exhibited the peak at 0.55 V. However, the shoulder at $0.40 \mathrm{~V}$ observed for the $\left(\mathrm{HgCl}_{4}\right)^{2-}$ solution (Fig. 10(b)) disappeared. Since the peaks $0.40 \mathrm{~V}$ and $0.55 \mathrm{~V}$ can be assigned to $\mathrm{Hg} \rightarrow \mathrm{Hg}^{+}+\mathrm{e}^{-}$and $\mathrm{Hg}^{+} \rightarrow \mathrm{Hg}^{2+}+\mathrm{e}^{-}$, respectively, the disappearance of the former may be caused by the disproportionation, $\mathrm{Hg}^{2+}+\mathrm{Hg} \rightarrow \mathrm{Hg}_{2}{ }^{2+}$, which facilitates formation of $\mathrm{Hg}_{2}{ }^{2+}$ without electrochemical oxidation. The peak current at $0.55 \mathrm{~V}$ was proportional to the concentration of dispersed mercury for less than $2 \mu \mathrm{M}$, of which proportional constant was $3.0 \mathrm{~A} \mathrm{M}^{-1}$.

A mercury drop was inserted into $0.1 \mathrm{M} \mathrm{HClO}_{4}+0.05 \mathrm{M} \mathrm{NaCl}$ deaerated solution, which was left quiescently for $15 \mathrm{~h}$. An aliquot $2 \mathrm{~cm}^{3}$ over the mercury drop was suck out, and transferred to $10 \mathrm{~cm}^{3} 0.1 \mathrm{M} \mathrm{HClO}_{4}+0.05 \mathrm{M} \mathrm{NaCl}$ deaerated solution. The square-wave voltammogram after the cathodic deposition is shown in Fig. 11. From the 
peak current, we determined the concentration of spontaneously dispersed mercury to be $1.5 \mu \mathrm{M}$. The secondly sampled aliquot showed $1.2 \mu \mathrm{M}$. Iterative sampling obviously mixes the solution to decrease the concentration. DLS was applied to the same solution to yield kcps values, corresponding to $1.3 \mu \mathrm{M}$. Ionic strength increases surface tension to suppress emulsification.

Dispersed mercury droplets are predicted to diffuse to the electrode to be oxidized, like the behavior of redox molecules with the reduced state. Although cyclic voltammetry in self-dispersed mercury suspensions was made at the platinum electrode, the oxidation wave could not be distinguished from the background unequivocally. We attempted to make voltammetry of the mercury suspension forced by ultrasonication, which had high concentration. The anodic wave showed unclear peak, being drawn out, probably because the oxidation may be controlled by contacting force of the droplets on the electrode, as has been demonstrate in the dispersed polyaniline-latex particles $[34,35]$. There are further complications such as blocking of the oxidation by oxidized mercury on droplet surfaces, partial reaction of big particles [36,37], and irreproducible currents by wide distribution of droplet size.

\section{Conclusions}

Mercury metal in contact with water disperses spontaneously in droplet form 0.3 $\mu \mathrm{m}$ in diameter. The concentration of the dispersed mercury ranges from 1 to $3 \mu \mathrm{M}$, depending on solution conditions if mercury were to take molecular form. The concentrations have been determined by DLS, TG and voltammetry, showing consistent values. Since they are ca. 1000 times larger than the standard of the environmental limit, caution should be paid to water which is used for inhibition of mercury vapor. The 
dispersed particles are not mercury oxide, mercury chlorides or ions but the reduced form (metal), as can be demonstrated by the redox potential of voltammograms.

\section{References}

[1] Z. Samec, T. Kakiuchi, in: H. Gerischer, C.W. Tobias (Eds.), Advances in Electrochemical Science and Engineering, vol. 4, VCH, Weinheim, 1990, p. 297.

[2] T. Kakiuchi, N. Tsujioka, S. Kurita, Y. Iwami, Electrochem. Commun. 5 (2003) 159.

[3] M. G. Freire, P. J. Carvalho, R. L. Gardas, I. M. Marrucho, L.M.N.B.F. Santos, J. A. P. Coutinho. J. Phys. Chem. B 112 (2008) 1604.

[4] Y. A. Shchipunov, O. Schmiedel, Langmuir 12 (1996) 6443.

[5] S. Pautot, B. J. Frisken, J.-X. Cheng, X. S. Xie, D. A. Weitz, Langmuir 19 (2003) 10281.

[6] H. Gonza'lez-Ochoa, L. Ibarra-Bracamontes, J. L. Arauz-Lara, Langmuir 19 (2003) 7837.

[7] J. Chen, M. Satoh, J. Electroanal. Chem. 572 (2004) 153.

[8] K. Aoki, M. Li, J. Chen, T. Nishiumi, Electrochem. Commn. 11 (2009) 239.

[9] M. Li, K. Aoki, J. Chen, T. Nishiumi, J. Electroanal. Chem. 655 (2011) 159.

[10] S. Sacanna, W. K. Kegel, A. P. Philipse, Langmuir 23 (2007) 10486.

[11] K. Maeda, S. Kihara, M. Suzuki, M. Matsui, J. Electroanal. Chem. 295 (1990) 183.

[12] Y. Shao, A.A. Atewart, H.H. Girault, J. Chem. Soc. Faraday Trans. 87 (1991) 2593.

[13] M. Tsionsky, A.J. Bard, M.V. Mirkin, J. Phys. Chem. 100 (1996) 17881.

[14] W. Hyk, Z. Stojek, J. Phys. Chem. B 102 (1998) 577.

[15] K. Aoki, M. Satoh, J. Chen, T. Nishiumi, J. Electroanal. Chem. 595 (2006) 103.

[16] T. Kakiuchi, Electrochem. Commun. 2 (2000) 317.

[17] M. Nakagawa, N. Sezaki, T. Kakiuchi, J. Electroanal. Chem. 501 (2001) 260. 
[18] K. Aoki, H. Toda, J. Yamamoto, J. Chen, T. Nishiumi, J. Electroanal. Chem. DOI 10.1016/j.jelechem.2012.01.013.

[19] J. Heyrovsky, J. Kuta, Principles of Polarography, Academic Press, New York, 1966, pp. 429-463

[20] R. N. O'Brien, F. P. Dieken, Can. J. Chem. 48 (1970) 2651.

[21] Md. M. Islam, T. Okajima, T. Ohsaka, J. Electroanal. Chem. 618 (2008) 1.

[22] M. C. Canela, W. F. Jardim, J. Brazil. Chem. Soc. 8 (1997) 421.

[23] D.Sarkar, Envir. Geo. 10 (2003) 151.

[24] E. Onat, J. Inorg. Nuclear Chem. 36 (1974) 2029.

[25] Yu.V. Zelyukova, T.O. Didorenko, Zh. Anal. Khim. 42 (1987) 1431.

[26] T. Okabe, J. Ferracane, C. Cooper, H. Matsumoto, M. Wagner, J. Dent. Res. 66 (1987) 33.

[27] M. Marek, J. Dent. Res. 69 (1990) 1167.

[28] Y. Takahashi, J. Hasegawa, Y. Kameyama, Dent. Mat. 5 (1989) 256.

[29] I. M. Lifshitz, V. V. Slyozov, J. Phys. Chem. Solids 19 ( 1961) 35.

[30] C. Wagner, Z. Electrochem. 65 (1961) 581.

[31] C. Wagner, Colloid Polymer Sci. 254 (1976) 400.

[32] K. Aoki, J. Colloid Interface Sci. 360 (2011) 256.

[33] L.G. Sillen and A.E. Martell, Stability Constants of Metal-Ion Complexes, pp. 8-6 to 8-11, The Chemical Society, London, 1964.

[34] K. Aoki, Q. Ke, J. Electroanal. Chem. 587 (2006) 86.

[35] Q. Ke , K. Aoki, J. Colloid Interface Sci. 305 (2007) 40.

[36] K. Aoki, Q. Ke, J. Electroanal. Chem. 587 (2006) 86.

[37] C. Xu, J. Chen, K.Aoki, Electrochem. Commun. 5 (2003) 506. 